\title{
VIVÊNCIAS E ESTÁGIOS NA REALIDADE DO SISTEMA ÚNICO DE SAÚDE: DEBATENDO GÊNERO, FEMINISMO E DIVERSIDADE
}

EXPERIENCES AND INTERNSHIPS IN THE REALITY OF THE BRAZILIAN NATIONAL HEALTH SYSTEM: DEBATING GENDER, FEMINISM AND DIVERSITY

EXPERIENCIAS Y PASANTÍAS EN LA REALIDAD DEL SISTEMA ÚNICO DE SALUD BRASILEÑO: DEBATIENDO GÉNERO, FEMINISMO Y DIVERSIDAD

Gilson Aquino Cavalcante ${ }^{1}$

Jonatas Gomes Neri 2

Sueli Alves Castanha ${ }^{3}$

Juciano de Sousa Lacerda ${ }^{4}$

Palavras-chave: Sistema Único de Saúde; Identidade de Gênero; Saúde Pública; Educação em Enfermagem.

Keywords:

Brazilian National Health System; Gender Identity; Public Health; Education, Nursing.

Palabras clave: Sistema Único de Salud Brasileño; Identidad de Género; Salud Pública; Educación en Enfermería.

Submetido: 19/09/2017

Aprovado: $18 / 04 / 2018$

Autor(a) para Correspondência: Gilson Aquino Cavalcante End: Rua Henrique Dias, 39 - G - Igapó - CEP: 59104-300, Natal-RN

E-mail: aquinogilson@yahoo.com.br

\section{RESUMO}

Este artigo descreve a experiência de estudantes de graduação em Enfermagem no programa "Vivências e Estágios na Realidade do Sistema Único de Saúde" (VER-SUS), versão 2016.1, que ocorreu em Natal (RN), e sua abordagem às temáticas gênero, feminismo e população de lésbicas, gays, bissexuais, travestis, transexuais e transgêneros (LGBT). Trata-se de um relato de experiência descrito pelos estudantes por meio de um portfólio entregue ao final das vivências, como forma de comprovar a participação e obter a certificação do programa. No decorrer de toda a vivência, os estudantes se depararam com os mais diferentes cenários $e$ realidades da saúde brasileira no que tange ao Sistema Único de Saúde (SUS), como as unidades básicas de saúde (UBS) e sua organização. Esse contato possibilitou a observação do desconhecimento dos estudantes sobre o SUS e sua organização, bem como acerca das temáticas gênero, feminismo e população LGBT. O VER-SUS constitui um importante dispositivo que possibilita aos estudantes participantes adentrar um espaço diversificado de aprendizagem e vivências relacionadas a seu futuro cotidiano de trabalho. Assim sugere-se a continuação e divulgação desse programa, que proporciona capacitação em diversas temáticas no que se refere a atenção à saúde e organização do sistema de saúde brasileiro.

1. Aluno de Graduação em Enfermagem no Centro Universitário Maurício de Nassau (Uninassau). Natal (RN), Brasil. E-mail: aquinogilson@yahoo.com.br

2. Aluno de Graduação em Enfermagem no Uninassau. Natal (RN), Brasil. E-mail: jonatasgomesneri@gmail.com 3. Enfermeira. Especialista em Saúde Coletiva e Saúde da Família. Instituto Superior e Centro Educacional Luterano "Bom Jesus" (IELUSC). Joinville (SC), Brasil. E-mail: sualves13@gmail.com

4. Doutor em Ciências da Comunicação. Professor na Universidade Federal do Rio Grande do Norte (UFRN). Membro do Núcleo de Estudos em Saúde Coletiva (Nesc) da UFRN. Coordenador do Laboratório de Pesquisa e Estudos em Comunicação Comunitária e Saúde Coletiva (Lapeccos) da UFRN. Natal (RN), Brasil. E-mail: juciano. lacerda@gmail.com 


\section{ABSTRACT}

This article describes the experience of undergraduate Nursing students in the program 'Experiences and Internships in the Reality of the Brazilian National Health System' (VER-SUS), version 2016.1, which took place in Natal, Rio Grande do Norte, Brazil, and its approach to the themes gender, feminism, and lesbian, gay, bisexual, and transgender (LGBT) population. This is an experience report described by students through a portfolio delivered when the experiences came to an end, as a way to prove participation and obtain certification from the program. Throughout the experience, students faced the most varied Brazilian health scenarios and realities regarding the Brazilian National Health System (SUS), such as Brazilian primary health centers (UBS) and their organization. This contact made it possible to observe the lack of knowledge on the SUS and its organization among students, as well as about the themes gender, feminism, and LGBT population. The VER-SUS is a significant device that enables participating students to get into a diversified space for learning and experiences related to their future everyday work. Thus, we suggest the continuation and dissemination of this program, which provides training in various themes concerning health care and organization of the Brazilian health system.

\section{RESUMEN}

Este artículo describe la experiencia de estudiantes de pregrado en Enfermería en el programa "Experiencias y Pasantías en la Realidad del Sistema Único de Salud Brasileño" (VER-SUS), versión 2016.1, que tuvo lugar en Natal, Rio Grande do Norte, Brasil, y su abordaje a los temas género, feminismo y población de lesbianas, gays, bisexuales y transexuales (LGBT). Este es un informe de experiencia descrito por los estudiantes a través de un portafolio entregado cuando las experiencias llegaron a su fin, como una forma de probar la participación y obtener la certificación del programa. A lo largo de la experiencia, los estudiantes se enfrentaron a los escenarios y las realidades más diversas de la salud brasileña con respecto al Sistema Nacional de Salud Brasileño (SUS), como los centros primarios de salud brasileños (UBS) y su organización. Este contacto permitió observar la falta de conocimiento acerca del SUS y su organización entre los estudiantes, así como sobre los temas género, feminismo y población LGBT. El VER-SUS es un importante dispositivo que permite a los estudiantes participantes adentrar en un espacio diversificado para el aprendizaje y las experiencias relacionadas con su futuro cotidiano de trabajo. Así, se sugiere la continuación y difusión de este programa, que brinda capacitación en diversos temas relacionados con atención a la salud y organización del sistema de salud brasileño.

\section{INTRODUÇÃO}

O Sistema Único de Saúde (SUS) é considerado um dos mais amplos sistemas públicos de saúde do mundo, pautando-se por um arcabouço de princípios e diretrizes. Idealizado na VIII Conferência Nacional de Saúde, realizada em 1986, foi instituído pela Constituição Federal de 1988, acatando as proposições do movimento da Reforma Sanitária Brasileira (RSB), que teve como principal bandeira a luta pela democratização da saúde. Para tanto, incorporou mudanças no papel do Estado no setor saúde, a fim de garantir acesso integral, universal e gratuito - o SUS garante atendimento a toda a população, independente de sexo, situação econômica, idade, cor da pele e orientação sexual. Para uma operação eficiente desse sistema de saúde, em consonância com seus princípios, além de gestão, são necessários trabalhadores capacitados em suas respectivas áreas. No entanto, com frequência se observa certo despreparo técnico, científico e político entre esses profissionais ${ }^{1-3}$.

Há significativa lacuna na formação e no preparo dos profissionais da saúde considerados qualificados para atuar nas diversas instâncias do SUS ${ }^{2-4}$. Romper com os modelos assistenciais hegemônicos se mostra um desafio constante, visto que as matrizes curriculares em saúde não atendem adequadamente as propostas do processo saúde-doença e seus determinantes. 0 ordenamento jurídico brasileiro assimilou a Teoria da Determinação Social no processo saúde-doença, nos termos da medicina social e da saúde coletiva. Contudo, atualmente não se observa valorização desse princípio por parte da sociedade e do Estado nem reconhecimento da "saúde da população como resultante das formas de organização social da produção"1:252. Ao analisar currículos de escolas de Enfermagem, pesquisadores observaram que o perfil dos profissionais continua voltado a modelos hospitalocêntricos - há sinalização de reorientação da formação, porém, trata-se de um processo lento ${ }^{2}$. 
Nessa mesma ótica, as abordagens da diversidade, do gênero e da sexualidade são pouco debatidas durante a formação em saúde; as discussões se restringem às funções e disfunções do sistema sexual e reprodutor. Na escola, os temas transversais sobre gênero e sexualidade ficam à margem dos debates e, quando entram em pauta, surgem dúvidas quanto à sua credibilidade e desqualificação dos profissionais/ docentes que buscam discuti-los ${ }^{5}$. No Ensino Superior, essas mesmas questões balizam uma formação que reafirma papéis sociais de gênero previamente estabelecidos. Michel Foucault caracteriza tal contexto como poder disciplinador da escola - que treina e silencia os corpos, constituindo o indivíduo necessário à manutenção do capital ${ }^{6}$.

Abordar gênero, sexualidade e diversidade nas várias especialidades do setor saúde, dentro e fora das instituições de formação, busca reafirmar a universalidade, integralidade e gratuidade no âmbito do SUS.

Com vistas a minimizar as lacunas na formação de profissionais do SUS, surgiu em 2002 o programa “Vivências e Estágios na Realidade do Sistema Único de Saúde" (VER-SUS) - projeto financiado pelo Ministério da Saúde (MS) e coordenado pela Rede Unida e pela União Nacional dos Estudantes (UNE), que incentiva o protagonismo estudantil ao organizar a imersão de estudantes de graduação no SUS, sejam estes do setor saúde ou de outros setores, interessados em estabelecer vivências durante o período de férias de instituições públicas e privadas ${ }^{7}$. 0 VER-SUS capacita e complementa a formação desses estudantes, pois discute diversas questões que permeiam todos os níveis de atenção à saúde no âmbito do SUS, dentre as quais se encontram as temáticas gênero, diversidade e feminismo.

Este artigo descreve a experiência de estudantes de graduação em Enfermagem no programa VER-SUS, versão 2016.1, que ocorreu em Natal (RN), com abordagem das temáticas gênero, feminismo e população de lésbicas, gays, bissexuais, travestis, transexuais e transgêneros (LGBT).

\section{METODOLOGIA}

Trata-se de relato de experiência de estudantes de graduação em Enfermagem, de instituição de Ensino Superior privada, no programa VER-SUS, versão 2016.1.

A vivência ocorreu em Natal, de 08/01/2016 a 19/01/2016, coincidindo com o período de férias da

\section{O VER-SUS \\ capacita \\ e complementa a \\ formação desses \\ estudantes...}

instituição em questão. Participaram do programa VER-SUS, versão 2016.1, cerca de 60 estudantes de graduação de diversas instituições públicas e privadas e de diferentes áreas do conhecimento, dentre as quais: Enfermagem, Medicina, Odontologia, Nutrição, Fisioterapia, Gestão de Políticas Públicas, Psicologia, Direito e Jornalismo. Esses participantes ficavam alojados durante o dia, dormiam e realizavam as refeições no centro de treinamento, com saída apenas para os locais das práticas de vivências.

Para organizar e desenvolver o projeto, os participantes foram divididos em 5 núcleos de base (NB), compostos por 10 viventes (estudantes) e 2 facilitadores (participantes de edições anteriores) selecionados para mediar as atividades com base na experiência adquirida.

A seleção para participar do programa foi realizada on-line, na plataforma Observatório de Tecnologias de Informação e Comunicação em Sistemas de Saúde (OTICS), conveniada ao MS ${ }^{8}$. 0 formulário de inscrição consistia em um questionário sobre as variáveis sociodemográficas e acadêmicas. Os critérios de inclusão foram: a) disponibilidade de participação em tempo integral; e b) estar matriculado e frequentando curso de graduação em instituição de Ensino Superior pública ou privada.

A discussão a seguir resultou dos relatos de viventes que participaram do VER-SUS, versão 2016.1, em Natal. Tais relatos foram produzidos no final de cada dia de vivência, por meio dos debates entre os $N B$, onde eram colocadas as vivências e percepções do dia. No fim das vivências, todos os relatos de cada vivente foram enviados, por meio de um portfólio, na plataforma 0TICS; a visualização ficou restrita aos viventes de todos os NB e à coordenação. Essa é uma forma de compartilhar as experiências entre os viventes, além de comprovar a participação e obter a certificação do programa.

\section{RESULTADOS E DISCUSSÃO}

No decorrer de toda a vivência, os estudantes se 
depararam com os mais diversos cenários e realidades da saúde brasileira no que tange ao SUS, como as unidades básicas de saúde (UBS) e sua organização. Esse contato possibilitou a observação do desconhecimento dos estudantes sobre o SUS e sua organização, bem como acerca das temáticas gênero, feminismo e população LGBT.

Após cada dia de vivência, os viventes organizavam rodas de conversa e confeccionavam relatórios dos principais pontos de cada instância ou formação no projeto. Para contribuir com as discussões e enriquecer a capacitação, também contavam com recursos textuais, audiovisuais e lúdicos, como filmes, pintura, artesanato e dramatização. Todas essas tarefas eram executadas com o apoio dos facilitadores.

Quando foi abordada a temática gênero, percebeuse que os estudantes desconheciam o atual conceito de gênero, bem como as características e as necessidades específicas de cada grupo. Inclusive as concepções acerca do feminismo, como movimento social e cultural, e da população LGBT.

\section{Gênero}

0 projeto em si foi todo pautado pela temática gênero. Desde a seleção dos participantes, a comissão responsável buscou garantir a igualdade do número de participantes entre mulheres, homens e população LGBT.

Diante do desconhecimento dos viventes do VERSUS sobre o referido tema foram realizadas oficinas de formação, com o propósito de esclarecer conceitos e promover debates e discussões acerca dessas questões. Foram disponibilizados textos sobre conceituação de gênero para cada NB e no final do dia houve uma reunião com todos os participantes, com vistas a promover capacitação voltada às diversas definições de gênero, estabelecido em 1993 como elemento constitutivo de relações sociais fundadas sobre as diferenças percebidas entre os sexos - sendo o gênero o primeiro modo de atribuir significado às relações de poder ${ }^{9}$. E em 2011, o MS definiu gênero como a construção histórica, cultural e política das diversas possibilidades do ser feminino e/ou masculino. Ultrapassa, portanto, o "ser macho" ou "ser fêmea", originando diferentes papéis e funções sociais, cuja consequência é a distribuição desigual do poder e das oportunidades a partir do que é definido social e culturalmente como "coisa de homem" ou "coisa de mulher"10.

\section{As vivências buscaram a compreensão e o diálogo com o movimento feminista...}

Assim, os viventes foram expostos às atuais particularidades da diversidade, principalmente no que tange às necessidades e aos direitos da população feminina e da população LGBT. Esses debates objetivam preparar os viventes para o contato direto com a diversidade como profissionais das diversas áreas, proporcionando um cuidado livre de preconceito.

\section{Feminismo}

No percurso do programa VER-SUS, versão 2016.1, em Natal, reservou-se um período para o debate sobre a temática do feminismo. Em um primeiro momento surgiu um espaço misto, com a presença de homens e mulheres, onde foi abordada a coparticipação da população masculina no movimento feminista. Posteriormente, surgiu um espaço auto-organizado, composto somente por mulheres, que tratou de versar sobre as dificuldades vivenciadas pelas mulheres na sociedade e a luta pela garantia de direitos igualitários.

Durante o período de vivência ocorreram diversas intervenções, uma delas por meio de frases colocadas em cima das mesas no refeitório que explicitavam atitudes que fortalecem condutas machistas, ainda disseminadas pela sociedade na vida cotidiana.

As vivências buscaram a compreensão e o diálogo com o movimento feminista, que tem como interesse central a igualdade de direitos entre homens e mulheres, questionando a subordinação à qual as mulheres são submetidas ${ }^{11}$. Com base nisso, a comissão político-pedagógica do projeto garantiu a biparidade de gênero durante a realização das atividades, de modo que todos os diálogos ocorreram com proporcionalidade entre homens e mulheres.

Muitos dos viventes não conheciam o conceito e a origem desse movimento, principalmente em relação a ser um movimento social e cultural evidenciado em meados dos anos 1960, em resposta à opressão 
enfrentada ao longo dos tempos pelas mulheres.

Observou-se que, além da falta de informação, ainda há resistência em relação ao termo feminismo, colocado como sinônimo de machismo e não discutido em sua essência. Desse modo, os momentos vivenciados no âmbito desse tema se mostraram de suma importância para a desconstrução e a desnaturalização dos papéis que distinguem homens e mulheres, marcadores sociais que geram desigualdades e vulnerabilidades e permeiam um conjunto de determinantes que interferem no processo de viver, adoecer e morrer das diversas populações.

\section{População LGBT}

Seguindo o mesmo método trabalhado no feminismo, no espaço misto foram debatidas as especificidades da população LGBT e o enfrentamento das dificuldades que ela enfrenta na sociedade, principalmente quando relacionadas ao acesso a serviços de saúde. Esse tema foi trabalhado por meio da dramatização do uso do nome social no SUS e da terapia hormonal para adequação corpórea à identidade de gênero. Assim, essas estratégias têm o intuito de capacitar os estudantes para que, em sua prática profissional, tratem todas as pessoas de modo livre de preconceitos e estigmas.

A dramatização se mostrou importante por promover o debate da experiência pessoal relatada por cada indivíduo presente. Como, por exemplo, o caso de um dos viventes que havia sido expulso de casa depois de assumir sua homossexualidade. Ele recebeu apoio dos demais, o que serviu para promover o companheirismo no âmbito do programa.

Todas as questões abordadas tiveram a finalidade de garantir o direito constitucional da universalidade do SUS. Mostrou-se possível promover a saúde integral da população LGBT, sem discriminação e preconceito institucional, contribuindo para a redução das desigualdades e a consolidação do SUS nos termos da Política Nacional de Saúde da População LGBT ${ }^{12}$.

\section{CONCLUSÃO}

0 VER-SUS constitui um importante dispositivo que possibilita aos estudantes participantes adentrar um espaço diversificado de aprendizagem e vivências relacionadas a seu futuro cotidiano de trabalho.

Nos tempos atuais, constata-se a necessidade de reorientar a formação dos profissionais da saúde e modificar as grades curriculares no setor saúde, com

\section{...promover a saúde integral da população LGBT, sem discriminação $e$ preconceito institucional...}

a retirada de disciplinas das áreas de Ciências Humanas e a oferta de cursos na modalidade educação a distância. Isso contribui para a manutenção da lacuna entre formação e preparo e não favorece o estabelecimento de diálogos que busquem a compreensão das doenças que acometem a população.

Nesse sentido, o programa VER-SUS oferece um ambiente ímpar para a formação social e política de futuros profissionais da saúde. Considerando que, em muitas realidades, a depender da grade curricular e da missão da instituição de Ensino Superior, os profissionais da saúde são formados no SUS (por meio de estágios obrigatórios), porém, não são formados para o SUS, a formação desses profissionais demanda avanços para atender às concepções dispostas nas bases legais do SUS, que permeiam as necessidades e as práticas de saúde em todo o país.

0 programa VER-SUS deve seguir promovendo a formação de profissionais comprometidos ética e politicamente com as demandas de saúde das populações atendidas por eles - como aquelas permeadas pela temática gênero, com base na Política Nacional de Saúde da População LGBT, tendo em vista que o preconceito é um determinante social do adoecimento nessa população.

Assim, sugere-se a continuação, a divulgação e o fortalecimento desse programa, que proporciona capacitação em diversas temáticas da atenção e do cuidado no âmbito do sistema de saúde brasileiro.

\section{CONTRIBUIÇÃO DOS AUTORES}

Gilson Aquino Cavalcante contribuiu com a concepção do estudo e a preparação do manuscrito. Jonatas Gomes Neri contribuiu com a concepção do estudo e a preparação e estruturação do manuscrito. Sueli Alves Castanha contribuiu com a redação e a revisão crítica do manuscrito. Juciano de Sousa Lacerda contribuiu com a revisão crítica do manuscrito. 


\section{REFERÊNCIAS}

1. Paim JS. Reforma sanitária brasileira: contribuição para a compreensão e crítica. 2007. Tese (Doutorado em Saúde Pública) - Instituto de Saúde Pública, UFBA, Bahia. Available from: https://repositorio.ufba.br/ri/bitstream/ ri/10376/1/5555555555.pdf

2. Silva RPG, Rodrigues RM. Sistema Único de Saúde e a graduação em enfermagem no Paraná. Rev Bras Enferm [serial on the internet]. 2010 [cited 2018 Apr 22];63(1):6672. Available from: https://pdfs.semanticscholar.org/8728/ 5761bf2a5165d15c0955a6471af914952b68.pdf

3. Gomes AMT, Oliveira DC, Sá CP. As representações sociais do Sistema Único de Saúde no município do Rio de Janeiro. Rev Latinoam Enferm [serial on the internet]. 2008 [cited 2018 Apr 22];16(1):122-9. Available from: http://www. scielo.br/pdf/rlae/v16n1/pt_18

4. Lira Neto JCG, Freitas RWJF, Brito ECC, Santos LR, Alves LEP, Alves LRA. VER-SUS: an experience report about a life experience-stage in the reality of the Unified Health System. Rev Enferm UFPE On Line [serial on the internet]. 2013 [cited 2018 Apr 22];7(Spec):1042-6. Available from: https://periodicos.ufpe.br/revistas/revistaenfermagem/ article/viewFile/11574/13572

5. Leal CJ, Oliveira LB. A escola e a desnaturalização do gênero e da sexualidade: na construção de uma sociedade de respeito às diferenças. In: Bento $B$, Félix-Silva $A V$, organizer. Desfazendo gênero: educação da diferença, masculinidades, feminismos e literatura. Natal: Ed. UFRN; 2015. p. 21-46.

6. Gore JM. Foucault e educação: fascinantes desafios. In: Silva TT, organizer. 0 sujeito da educação: estudos foucaultianos. 7. ed. Petrópolis (RJ): Vozes; 2010. p. 9-20.

7. Pedrosa R, Ferla AA. Refletindo o Programa Mais Médicos em Novo Hamburgo, Rio Grande do Sul, a partir do olhar dos participantes do VER-SUS, edição verão 2014. IV Congresso Piauiense de Saúde Pública; 2015; Parnaíba. Anais. (Sanare (Sobral, Online) [serial on the internet]. 2015 [cited 2018 Apr 22];14(Suppl 1):42). Available from: https://sanare. emnuvens.com.br/sanare/article/viewFile/666/369

8. VER-SUS [homepage on the internet]. Porto Alegre: Ministério Saúde; [s.d] [cited 2018 Apr 22]. Available from: http://www.otics.org/estacoes-de-observacao/versus

9. Oliveira EN, Araújo MFM. Gênero e saúde. Sanare (Sobral, Online) [serial on the internet];2002 [cited 2017 May 4];3(1):59-62. Available from: http://sanare.emnuvens. com.br/sanare/article/view/90/82

10. Brasil. Diversidades sexuais. Brasília (DF): Ministério da Saúde; 2011.

11. Levatti EG. Um breve olhar acerca do movimento feminista. Bauru (SP): Unesp; 2011.
12. Brasil. Política Nacional de Saúde Integral de Lésbicas, Gays, Bissexuais, Travestis e Transexuais. Brasília (DF): Ministério da Saúde; 2012.
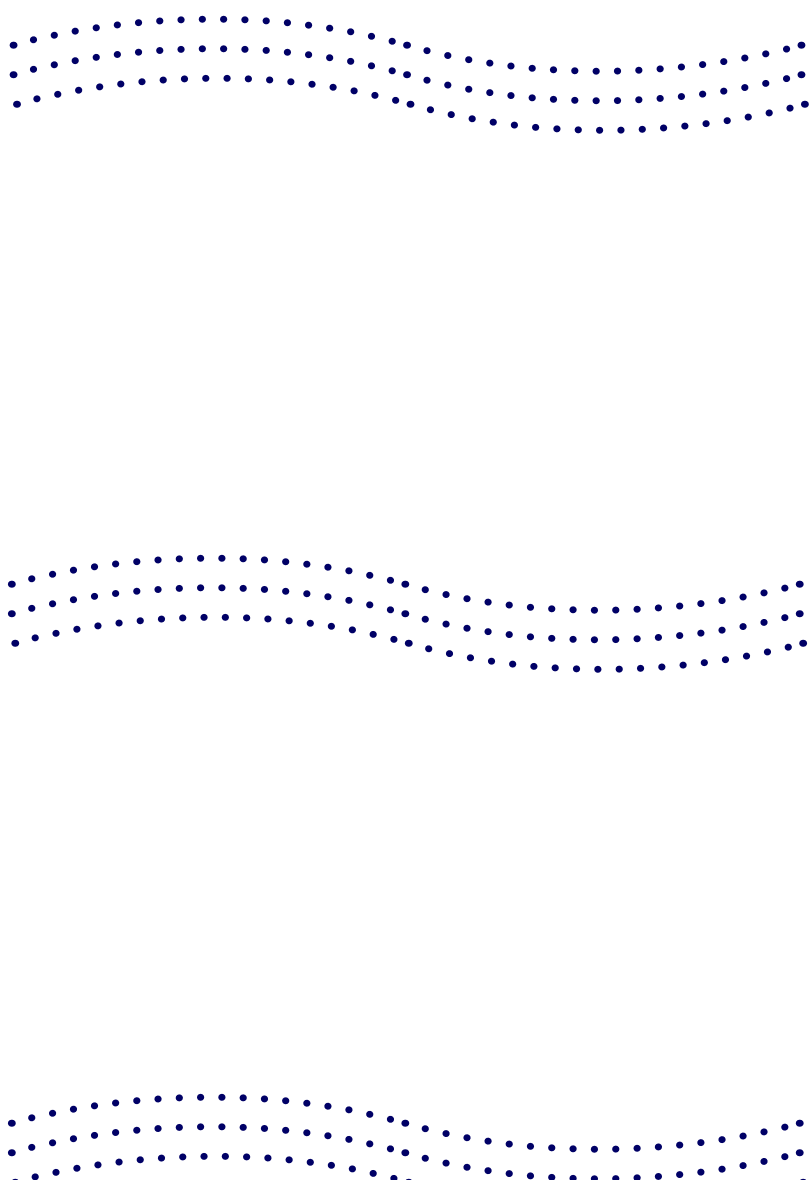
$\ldots \ldots \ldots \ldots \ldots \ldots \ldots \ldots$

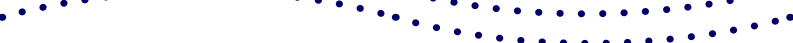

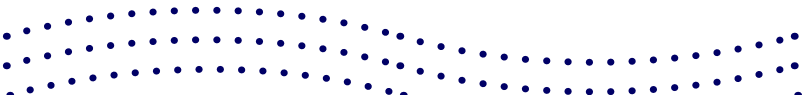
$\ldots \ldots \ldots \ldots \ldots \ldots$ ............................ 